\title{
Aplicação de processo adsortivo para dessulfurização de combustíveis utilizando fibra de coco como adsorvente
}

\section{Application of adsorptive process for desulphursation of fuel using coconut fiber as adsorbents}

\author{
Jaqueline de Lima Nascimento ${ }^{1}$, Guilherme Augusto Magalhães Júnior ${ }^{1}$, \\ Rafael Ribeiro Portela ${ }^{1}$, Vicente de Oliveira Sousa Neto ${ }^{2}$, \\ Patrícia Marques Carneiro Buarque ${ }^{1}$, Mayara de Sousa Oliveira ${ }^{1}$ \\ Cícero Pessoa de Moura ${ }^{1}$
}

\footnotetext{
${ }^{1}$ Grupo de Analítica e Materiais - GAMa/IFCE: 63.902-580, Quixadá, Ceará, Brasil.

${ }^{2}$ Faculdade de Educação, Ciências e Letras do Sertão Central - FECLESC/UECE, CEP: 63900-000, Quixadá, Ceará, Brasil.

e-mail: jaquelinelima96@gmail.com, guilhermejr@ifce.edu.br, rafael.if.portela@gmail.com,pmcbuarque@gmail.com mdsoliveira24@gmail.com, ciceropessoa@ifce.edu.br vicente@uece.br
}

\section{RESUMO}

A presença de compostos orgânicos contendo enxofre nos combustíveis é um dos contribuintes da poluição atmosférica, pois quando entram em combustão nos motores, emitem poluentes derivados do enxofre, como os óxidos $\left(\mathrm{SO}_{\mathrm{x}}\right)$, que afetam negativamente a saúde humana e o meio ambiente. A remoção do excesso de enxofre presente na gasolina pode ser realizada pelo processo de adsorção, em que um material adsorvente captura o composto de enxofre de modo seletivo.

O desenvolvimento de adsorventes mais eficientes e de baixo custo de produção para a remoção de compostos sulfurosos tem contribuído, por exemplo, nos processos referentes ao refino do petróleo. A eficiência da dessulfurizarão está correlacionada às características apresentadas pelo adsorvente como: a capacidade de adsorção, durabilidade e regenerabilidade. Esse estudo tem como finalidade o preparo da fibra do coco impregnada com níquel para avaliar a sua capacidade adsortiva frente a compostos sulfurosos como o tiofeno presente nos combustíveis. O adsorvente utilizado foi a fibra do coco (mesocarpo) obtido na cidade de Quixadá/CE e o adsorbato utilizado foi o tiofeno, em solução de n-hexano.

Em teste de caracterização do adsorvente, observou-se que a quantidade de cargas protônicas na superfície da fibra in natura e na fibra modificada é função do pH. Os materiais apresentaram capacidade adsortiva de 35,0 $\mathrm{mg} . \mathrm{g}^{-1}$ para o composto sulfuroso. No estudo de equilíbrio, isoterma, foi melhor representado pelo modelo matemático de Redlich-Peterson, indicando heterogeneidade nos sítios de adsorção. A cinética de adsorção se mostrou mais adequada com o modelo de pseudo-segunda ordem.

Palavras-chave: Combustíveis, Enxofre, Fibra de coco

\section{ABSTRACT}

The presence of organic compounds with sulfur in fuels is one of the reasons for atmospheric pollution, since they liberate pollutants derived from sulfur such as oxides $\left(\mathrm{SO}_{\mathrm{X}}\right)$ when combusted in engines, which can affect both human health and the environment in a negative way. Removal of excessive sulfur from gasoline can be achieved through the process of adsorption, in which an adsorptive material captures the sulfur compound selectively.

The development of more efficient and low-cost adsorbents to remove sulfur compounds has been improving, for instance, processes used in oil refining. Efficiency of desulfurization is correlated to the characteristics found in the adsorbent such as adsorption capacity, durability and regenerateness. This study aims to prepare coconut fiber impregnated with nickel and evaluate its adsorptive capacity of sulfur compounds such as the thiophene present in fuels. The utilized adsorbent was coconut fiber (mesocarp) obtained in the city of 
Quixadá, state of Ceará, and the adsorbate was thiophene in n-hexane solution.

During an adsorbent characterization test it could be observed that the number of proton charges in the surface of both natural and modified fibers is a function of $\mathrm{pH}$. The materials showed adsorptive capacity of $35,0 \mathrm{mg} \cdot \mathrm{g}^{-1}$ of the sulfur compound. In the balance study, isotherm was best represented by the math model of Redlich-Peterson, which indicates heterogeneity in the adsorption sites. Adsorption kinetics was more adequate with the pseudo-second order model.

Keywords: Fuels, Sulfur, Adsorption

\section{INTRODUÇÃO}

A presença de compostos orgânicos contendo enxofre nos combustíveis é um dos grandes contribuintes da poluição atmosférica, principalmente após a combustão em que são emitidos poluentes $\mathrm{SO}_{\mathrm{x}}$ que afetam negativamente a saúde humana e o meio ambiente [1].

No Brasil, a partir de $1^{\circ}$ de janeiro de 2014 ocorreu uma alteração no teor médio de enxofre permitido na gasolina, que no ano de 2013 era de 200 ppm, agora essa concentração é de no máximo 50 ppm. Com essa ação da Agência Nacional do Petróleo, Gás Natural e Combustível (ANP), a qualidade da gasolina se assemelha a comercializada nos Estados Unidos, Canadá e Europa e as emissões de poluentes na atmosfera teve redução que contribui para uma melhoria da qualidade do ar [2].

Além de sua forma elementar, o enxofre também se encontra nos combustíveis como mercaptanas, sulfetos, tiofeno, benzotiofeno, dibenzotiofeno e seus derivados alquílicos. Esses compostos possuem alto ponto de ebulição e são dificilmente removidos por processos convencionais de tratamento [3]. A figura 1 mostra a estrutura molecular do composto tiofeno.

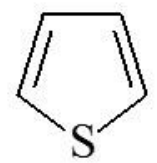

Figura 1: Estrutura molecular do tiofeno.

Para remoção do excesso de enxofre presente na gasolina, fazem-se uso de diversos processos, dentre os quais a adsorção. Nesse método, um material adsorvente é utilizado na captura seletiva de moléculas, adsorbado, dissolvidas em um meio [4]. O desenvolvimento de adsorventes mais eficientes na remoção de compostos sulfurosos tem contribuído no refino do petróleo, principalmente, em sua redução de custo, haja visto que os materiais adsorventes são eficientes, têm baixo preço de produção e facilidade de regeneração em determinados casos $[5,6]$.

A eficiência da dessulfurização está correlacionada às características apresentadas pelo adsorvente, como a sua capacidade de adsorção, durabilidade e regenerabilidade [7]. O fenômeno de adsorção é definido como o enriquecimento de um ou mais componentes em uma região interfacial devido a um não balanceamento de forças. Os principais elementos da adsorção são o fluido, a superfície (normalmente um sólido poroso) e os componentes retidos pela superfície, conforme figura $2 \mathrm{~A}$. O adsorvente é o sólido no qual ocorrerá a adsorção; o fluido em contato com o adsorvente é chamado de adsortivo, que pode ser uma fase gasosa ou uma solução líquida e adsorbato é a fase constituída pelos componentes retidos pelo adsorvente [8].

A interação entre o adsorbato e a superfície do adsorvente resulta da existência de forças atrativas não compensadas na superfície do sólido. Conforme a natureza das forças de ligação envolvidas, a adsorção pode ser classificada em: adsorção física (fisissorção) e adsorção química (quimissorção) $[4,9,10]$. Na fisissorção, as forças atrativas que ocorrem entre o adsorvente e o adsorbato são relativamente fracas, envolvendo, principalmente, interações de Van der Waals. Já na quimissorção, a interação entre a substância adsorvida e o sólido adsorvente é muito mais intensa, quase tão forte quanto a de uma ligação química $[4,10]$.

O processo de adsorção é convencionalmente descrito como ocorrendo em 4 (quatro) fases: na primeira fase, tem-se o transporte do analito do seio da solução à superfície limite, uma porção da fase fluida estagnada junto ao limite do adsorvente; a passagem por essa camada limite do fluido é a segunda fase do processo, figura 2B. Esses dois momentos da adsorção são referidos como transferência de massa externa, cuja resistência á mobilidade do analito é dada, quantitativamente, pelo coeficiente de transferência de massa $\left(\mathrm{k}_{\mathrm{f}}\right)$, em mg.h ${ }^{-1}$ [11]. As fases seguintes do mecanismo de adsorção são: transporte do soluto entre os poros da partícula do material adsorvente (fase 3); e a interação do analito com os sítios de adsorção do adsorvente (fase 4), figura 2B. 
A

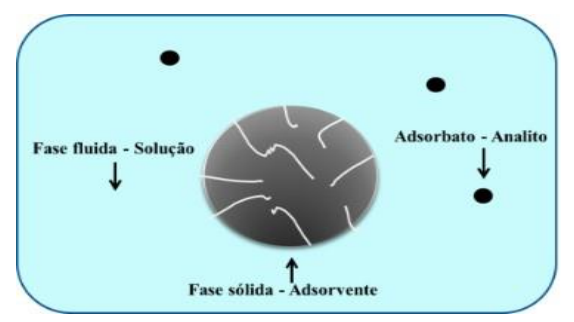

B

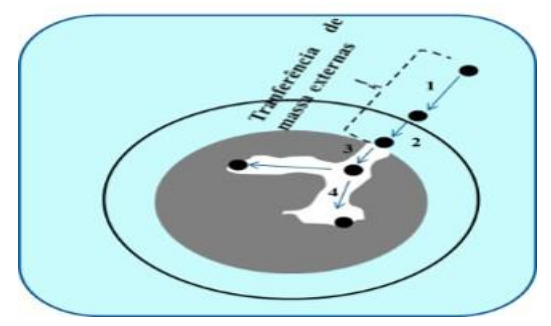

Figura 2: Representação proposta das etapas de adsorção. Fonte: Autor

A casca de coco é uma matéria-prima interessante como precursor de adsorvente devido a sua estrutura rica em carbono e baixo teor de cinzas, quando calcinada. Carbono ativado derivado da casca do coco é muito utilizado na remoção de diversificados agentes químicos orgânicos e inorgânicos. Zhao e pesquisadores o utilizou no estudo de adsorção, em fase gasosa, de tolueno e clorobenzeno [12]. Já Karri e colaboradores empregou o carbono ativado e modificado com $\mathrm{H}_{2} \mathrm{SO}_{4}$ na remoção de fenol de efluentes aquosos [13]. Já a fibra de coco in natura foi utilizada por Chwastowski e pesquisadores na remoção de cromo hexavalente de solução aquosa [14] e por, Aljeboree e colaboradores, no tratamento de efluente contaminados com corantes industriais [15].

Experimentalmente, existem alguns métodos utilizados no estudo da adsorção de analitos dissolvidos em solução, notadamente, solução aquosa, em adsorvente sólido. Dentre esses, destacam-se o método em solução fixa (batelada) e o de leito fixo (adsorção em coluna). Neste contexto, esse trabalho visa apresentar o potencial da fibra do coco in natura e modificada na remoção de compostos sulfurosos presentes em combustíveis via adsorção por batelada.

\section{MATERIAIS E MÉTODOS}

Uma amostra (30 g) da fibra do coco (mesocarpo) in natura foi colocada na estufa à $130{ }^{\circ} \mathrm{C}$ por $24 \mathrm{~h}$ para remoção total da umidade. Parte dessa amostra, $15 \mathrm{~g}$, foi ativada com uma solução de ácido clorídrico $(\mathrm{HCl})$ 1,0 mol. $\mathrm{L}^{-1}$ sob agitação por $24 \mathrm{~h}$ com o intuito de eliminar matéria orgânica incorporada e melhorar a porosidade do material. Depois de sucessivas lavagens com água destilada, a amostra foi colocada em solução de cloreto de níquel $\left(\mathrm{NiCl}_{2}\right)$ 1,0 mol.L $\mathrm{L}^{-1}$ e deixada sob agitação por $24 \mathrm{~h}$, seguido de lavagem com água destilada e colocada para secar por três dias em temperatura ambiente, obtendo a fibra modificada.

Análise do material por termogravimetria (TGs) foram obtidos usando equipamento TG-50A SHIMADZU, utilizando $10 \mathrm{mg}$ de amostra colocada em cadinhos de alumínio e aquecidas à $10^{\circ} \mathrm{C} . \mathrm{min}^{-1} \mathrm{sob}^{\text {flu- }}$ xo contínuo de nitrogênio de na faixa de temperatura de $10{ }^{\circ} \mathrm{C}$ a $300{ }^{\circ} \mathrm{C}$.

Medidas de cargas protônicas das amostras $(3,0 \mathrm{~g})$ da fibra in natura e modificada foram pulverizadas e utilizadas no preparo de uma suspensão da fibra em solução de nitrato de sódio $\left(\mathrm{NaNO}_{3}\right)$ 0,02 mol.L $\mathrm{L}^{-1}$, na proporção de 2,0 g/100 mL, mantendo mistura sob agitação por 18 horas. Finalizado esse período, foi o medido do pH da suspensão. Após ajustados a pH 3,0 usando solução de $\mathrm{HCl}$ 0,1 mol.L ${ }^{-1}$, realizou-se a titulação com solução de $\mathrm{NaOH} 0,1$ mol. $\mathrm{L}^{-1}$ até $\mathrm{pH}$ 10. Os resultados dessa medida podem ser usados para quantificar as cargas de prótons superficiais em mol. $\mathrm{g}^{-1}(\mathrm{Q})$ usando a equação 01 [16].

$$
Q=\frac{\mathrm{C}_{\mathrm{A}}-\mathrm{C}_{\mathrm{B}}+\left[\mathrm{OH}^{-1}\right]-\left[\mathrm{H}_{3} \mathrm{O}^{+}\right]}{\mathrm{a}}
$$

Onde $\mathrm{C}_{\mathrm{A}}$ é a concentração do ácido forte em um dado ponto da titulação, $\mathrm{C}_{\mathrm{B}}$ é a concentração da base forte usada para ajustar o valor de $\mathrm{pH}$ inicial, $\left[\mathrm{OH}^{-}\right]$e $\left[\mathrm{H}_{3} \mathrm{O}^{+}\right]$são as concentrações dos íons hidroxilas e hidrônios definidas pelo valor de pH. A razão massa da fibra por volume da solução é especificado pelo parâmetro (a) na equação.

Os estudos de adsorção foram realizados usando solução sintética de combustível, obtido pela dissolução do composto sulforoso, o tiofeno, em n-octano. Um teste inicial foi realizado em solução de 50,0 ppm, em triplicata, na proporção de $0,1 \mathrm{~g}$ do adsorvente em $100 \mathrm{~mL}$ da solução, em erlenmeyer com tampa de vedação. Os sistemas foram mantidos em um agitador mecânico (tipo shaker) a $200 \mathrm{rpm}$, durante 60 minutos e temperatura ambiente $\left( \pm 25^{\circ} \mathrm{C}\right)$. 
As soluções foram analisadas em um Trace GC Ultra (Thermo Scientif) com Deterctor de Ionização por Chama (CG/FID) e coluna de fase reversa. As medidas foram realizadas utilizando-se as seguintes condições: injetor PTV (modo Split); temperatura do injetor de $250{ }^{\circ} \mathrm{C}$; temperatura do forno de $40{ }^{\circ} \mathrm{C}$ por 1 minuto, rampa de $20^{\circ} \mathrm{C} / \mathrm{min}$ até $150^{\circ} \mathrm{C}$ por 1 minto; FID a $200{ }^{\circ} \mathrm{C}$ e tempo de análise de 7 minutos.

Para cinética de adsorção, frascos anabólicos com tampas perfuráveis de $25 \mathrm{~mL}$ contendo $20 \mathrm{mg}$ do adsorvente foram dispersos em 5,0 mL de solução de tiofeno de concentração $5,0 \mathrm{mg} . \mathrm{L}^{-1}$ e sob agitação de $200 \mathrm{rpm}$ à temperatura ambiente $\left( \pm 25^{\circ} \mathrm{C}\right)$. Alíquotas foram analisadas em CG/FID para quantificação da concentração residual do tiofeno. As constantes de velocidade foram determinadas de acordo com os modelos cinéticos de pseudo-primeira ordem, pseudo-segunda ordem.

As isotermas de adsorção foram preparadas, usando frascos anabólicos com tampas perfuráveis de 100 $\mathrm{mL}$ contendo $200 \mathrm{mg}$ do adsorvente e $50,0 \mathrm{~mL}$ de solução de tiofeno em concentração variando de 4,0 a 50 $\mathrm{mg} . \mathrm{L}^{-1}$ e sob agitação de $200 \mathrm{rpm}$ à temperatura ambiente $\left( \pm 25^{\circ} \mathrm{C}\right)$ durante 2 horas. Após esse período, três alíquotas de $1,0 \mu \mathrm{L}$ de cada sobrenadante foram analisadas no CG/FID. A capacidade de adsorção do adsorvente, $\mathrm{Q}_{\mathrm{e}}$ (mg do tiofeno/g) foi determinada com base na diferença de concentração usando o balanço de massa de acordo com a equação 02 [4] . O equilíbrio de adsorção discutido e comparado com os modelos de isotermas de adsorção de Langmuir, Freundlich e Redlich-Peterson.

$$
\mathrm{Q}_{\mathrm{e}}=\frac{\left(\mathrm{C}_{0}-\mathrm{C}_{\mathrm{e}}\right) \times \mathrm{V}}{\mathrm{m}}
$$

\subsection{Modelos matemáticos das isotermas}

O modelo teórico de Langmuir assume que a adsorção ocorre em sítios específicos e homogêneos na superfície do adsorvente, responsável pela adsorção de apenas uma molécula, não podendo ocorrer nenhuma adsorção adicional. Além disso, o modelo propõe que o adsorvente tem capacidade de adsorção finita para o adsorvato e que as moléculas adsorvidas não interferem na adsorção de sítios vizinhos [17]. O modelo de Langmuir pode ser escrito na forma da Equação 2.

$$
\mathrm{Q}_{\mathrm{e}}=\frac{\mathrm{q}_{\operatorname{máx} \times \mathrm{K}_{\mathrm{Lx}} \mathrm{Ce}}}{1+\mathrm{K}_{\mathrm{L} \times \mathrm{Ce}} \mathrm{Ce}}
$$

Em que $\mathrm{C}_{\mathrm{e}}\left(\mathrm{mg} \cdot \mathrm{L}^{-1}\right)$ é a concentração de equilíbrio. O parâmetro $\mathrm{q}_{\text {máx }}\left(\mathrm{mg} \cdot \mathrm{g}^{-1}\right)$ está relacionado com a capacidade máxima de adsorção e $\mathrm{K}_{\mathrm{L}}$ (constante de Langmuir) (L.mg ${ }^{-1}$ ) é a razão entre a constante cinética de adsorção e constante cinética de dessorção [18].

A isoterma de Freundlich é caracterizada por uma equação empírica, aplicável a sistemas caracterizados por heterogeneidade. O modelo sugere que não há limite para a capacidade de adsorção, pois a quantidade adsorvida tende ao infinito, ou seja, não prevê a saturação [17]. A isoterma de Freundlich é apresentada pela Equação 04.

$$
\mathrm{Q}_{\mathrm{e}}=\mathrm{K}_{\mathrm{F} \times \mathrm{Ce}^{1 / n}}
$$

Onde, $\mathrm{K}_{\mathrm{F}}$ é a constante de Freundlich $\left(\left(\mathrm{mg} \cdot \mathrm{g}^{-1}\right)\left(\mathrm{L}_{\mathrm{mg}} \mathrm{mg}^{-1}\right)^{1 / \mathrm{n}}\right)$ e $1 / \mathrm{n}$ é o fator de heterogeneidade. A constante de Freundlich está relacionada à capacidade de adsorção do sólido e o fator $\mathrm{n}$ indica se o processo é ou não favorável. Valores de n compreendidos de 1 a 10 são indicativos de condições favoráveis ao processo de adsorção [19].

A isoterma de Redlich-Peterson é usada para representar o equilíbrio de adsorção em uma ampla faixa de concentrações e, devido a essa versatilidade, pode ser aplicada em sistemas homogêneos e heterogêneos. A equação 05 representa o modelo matemático de Redlich-Peterson [4].

$$
\mathrm{Q}_{\mathrm{e}}=\frac{\mathrm{K}_{\mathrm{R} \times \mathrm{C}_{\mathrm{e}}}}{1+\mathrm{a} \times \mathrm{C}_{\mathrm{e}}^{\mathrm{b}}}
$$


Nessa equação, $K_{R}\left(L \cdot \mathrm{mg}^{-1}\right)$, a (L.mg $\left.{ }^{-1}\right)$ e o expoente b são as constantes de Redlich-Peterson. O parâmetro b varia de 0 a 1 . Quanto mais próximo de zero, maior é o grau de heterogeneidade na superfície de adsorção. Sendo igual a unidade, o modelo é convertido ao de Langmuir.

Temkin propôs um modelo (Equações 06 e 07) em que são considerados os efeitos das interações indiretas entre as moléculas do adsorbato. O modelo de Temkin assume que: (i) o calor de adsorção de todas as moléculas diminui linearmente com a cobertura, devido às interações adsorbato-adsorvente; e (ii) a adsorção é caracterizada por uma distribuição uniforme de energias de ligação [17].

$$
\begin{aligned}
& \mathrm{Q}_{\mathrm{e}}=\mathrm{B}_{\mathrm{x}} \ln \mathrm{K}_{\mathrm{T}}+\mathrm{B}_{\mathrm{x}} \ln \mathrm{C}_{\mathrm{e}} \\
& \mathrm{B}=\frac{\mathrm{RT}}{\mathrm{b}^{\prime}}
\end{aligned}
$$

$\mathrm{K}_{\mathrm{T}}$ é a constante de equilíbrio de ligação (L.mg ${ }^{-1}$ ), b’ é o calor de adsorção, R é a constante universal dos gases $\left(8,314 \mathrm{~J} . \mathrm{K}^{-1} \cdot \mathrm{mol}^{-1}\right)$ e T é a temperatura (K) [20].

\section{RESULTADOS}

Conforme metodologias descritas, o material adsorvente (fibra de coco in natura e modificada) foi caracterizado por análises de perda de massa com o aquecimento, TG e DTGs e por titulação ácido-base para determinar o ponto isoeletrônico e quantificar as cargas protônicas das fibras. A figura 03 mostra os resultados das análises térmicas, TG e DTG.

TG

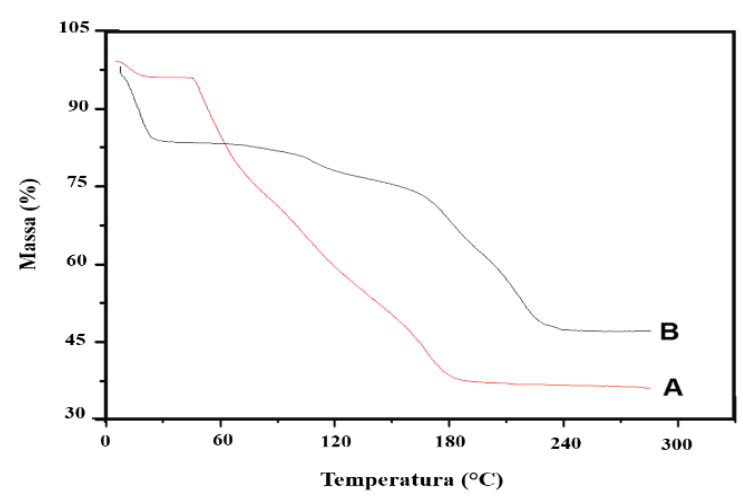

DTG

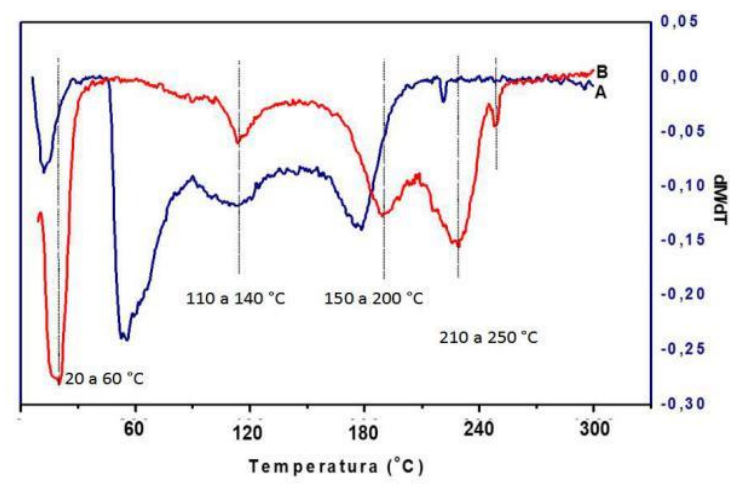

Figura 3: Termogramas (TG e DTG) da fibra in natura, curva A, e modificada, curva B. Fonte: Autor

As figuras 04 e 05 apresentam os gráficos resultantes da titulação ácido-base dos sólidos adsorventes.

A

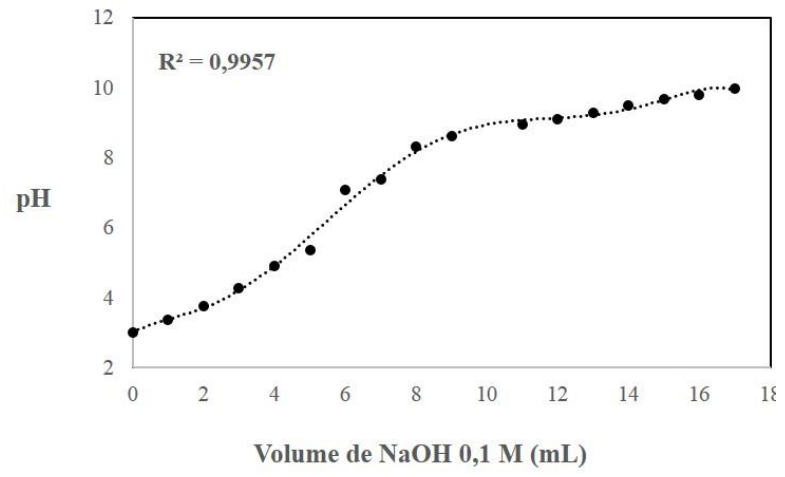

B

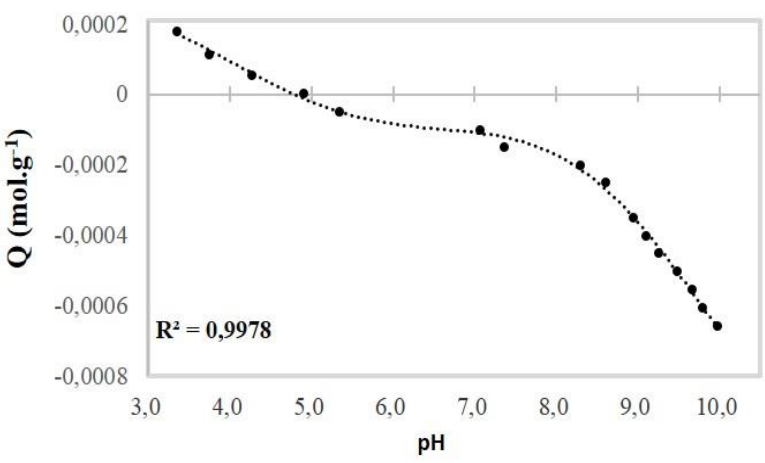

Figura 4: Curva de titulação (A) e a carga protônica (B) da fibra in natura. Fonte: Autor 


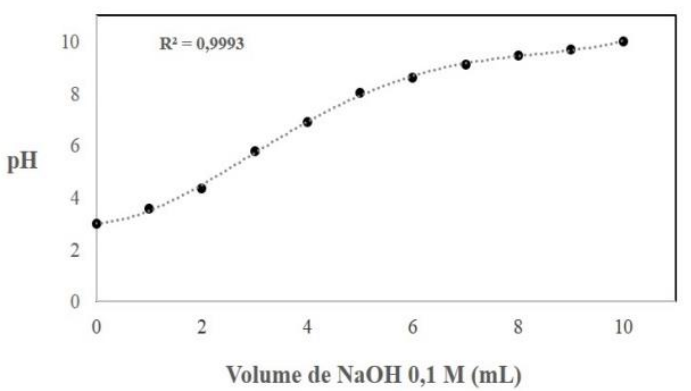

B

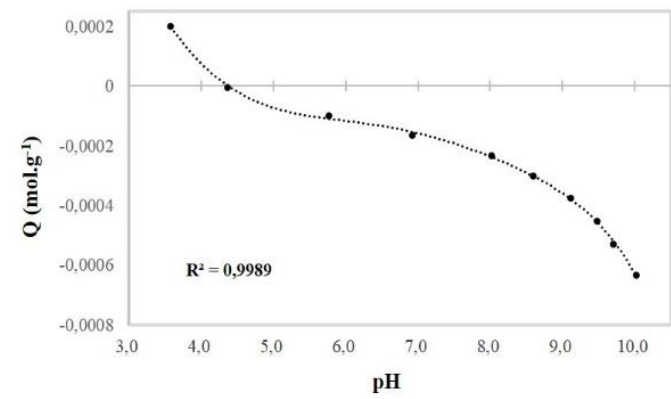

Figura 5: Curva de titulação (A) e a carga protônica (B) da fibra modificada. Fonte: Autor

No teste padrão de adsorção em batelada, após quantificação da concentração em CG/FID, verificouse no cromatograma (não mostrado), que o tempo de retenção do tiofeno é de 3,20 minutos. A capacidade de adsorção da fibra de coco natural e ativada são apresentadas na tabela 01.

Tabela 1: Dados do cromatograma relativo ao teste inicial de adsorção do tiofeno pela fibra de coco natural e ativada

\begin{tabular}{lcccccc}
\hline Amostra & Sistema & $\begin{array}{c}\text { Área } \\
\text { relativa }\end{array}$ & $\begin{array}{c}\text { Concentração } \\
\text { Final }(\mathbf{p p m})\end{array}$ & $\begin{array}{c}\text { Adsorção } \\
\mathbf{( \% )}\end{array}$ & $\begin{array}{c}\text { Qe } \\
\left(\mathbf{m g . \mathbf { g } ^ { - 1 }}\right)\end{array}$ & $\begin{array}{c}\mathbf{S} \\
(\mathbf{m g . g})\end{array}$ \\
\hline 1 & Controle & 1 & 50,0 & - & - & - \\
2 & Fibra natural & 0,286 & 14,3 & 71,4 & 35,7 & 0,007 \\
3 & Fibra ativada & 0,282 & 14,1 & 71,8 & 35,9 & 0,004 \\
\hline
\end{tabular}

A figura 06 mostra as curvas de cinética de adsorção relacionando a quantidade adsorvida no equilíbrio $\left(\mathrm{Q}_{\mathrm{e}}\right)$ versus tempo $(\mathrm{t})$ de adsorção do tiofeno pelos adsorventes (fibra in natura, curva $\mathrm{A}$, e a fibra modificada, curva B). Os dados experimentais foram aplicados aos modelos aos modelos de cinética de adsorção de pseudo-primeira ordem (PPO) e pseudo-segunda ordem (PSO), equações 08 e 09, respectivamente:

$$
\begin{aligned}
& \ln \left(Q_{e}-Q_{t}\right)=\ln Q_{e}-k_{1} t \\
& \frac{t}{Q_{t}}=\left(\frac{1}{Q_{e}{ }^{2} k_{2}}\right)+\frac{1}{Q_{e}} t
\end{aligned}
$$

Nessas expressões $\mathrm{k}_{1}\left(\mathrm{~min}^{-1}\right.$, $)$ e $\mathrm{k}_{2}\left(\mathrm{~g} \cdot \mathrm{mg}^{-1} \cdot \mathrm{min}^{-1}\right)$ são as constantes de velocidade e $\mathrm{Q}_{\mathrm{e}}$ representa a concentração de equilíbrio do adsorbato $\left(\mathrm{mg}^{-g^{-1}}\right)$ e $\mathrm{Q}_{\mathrm{t}}$, a concentração $\left(\mathrm{mg}^{\mathrm{g}} \mathrm{g}^{-1}\right)$ de adsorbato no tempo, $\mathrm{t}$ (min) [21]. Gráficos de linearização foram usados para obter os valores das constantes de velocidade de adsorção do tiofeno pela fibra in natura e modificada, assim como a concentração de equilíbrio do adsorbato. Observados nas tabelas 02 e 03 . O modelo que melhor descreveu o processo foi o de PSO. Nas tabelas 02 e 03 são mostrados os parâmetros cinéticos de adsorção. Verifica-se na figura 06 a boa concordância entre os resultados teóricos e os dados experimentais quando se aplica o modelo de PSO.

Tabela 2: Parâmetros da cinética de pseudo-primeira ordem (PPO): constante cinética $\left(\mathrm{k}_{1}\right)$, coeficientes de determinação $\left(\mathbf{R}^{2}\right)$ e capacidade de adsorção $\left(\mathbf{Q}_{\mathbf{e}}\right)$. Concentração do tiofeno: $5 \mathrm{mg} . \mathrm{L}^{-1}$ massa do adsorvente de $200 \mathrm{mg}$.

\begin{tabular}{ccccc}
\hline Fibra & $\mathbf{k}_{\mathbf{1}}(\mathbf{m i n})^{-\mathbf{1}}$ & $\mathbf{R}^{\mathbf{2}}$ & $\begin{array}{c}\mathbf{Q}_{\mathbf{e}}(\mathbf{c a l}) \\
\left(\mathbf{m g}^{\mathbf{- 1}}\right)\end{array}$ & $\begin{array}{c}\mathbf{Q}_{\mathbf{e}}(\mathbf{e x p}) \\
\left(\mathbf{m g . g}^{-\mathbf{1}}\right)\end{array}$ \\
\hline $\begin{array}{c}\text { In natura } \\
\text { Modificada }\end{array}$ & 0,0364 & 0,9876 & 43,86 & 35,63 \\
\hline
\end{tabular}

Comparando-se o valor de $\mathrm{k}_{2}$ da tabela 03 observa-se que $\mathrm{k}_{2}$ (amostra in natura) $<\mathrm{k}_{2}$ (amostra modificada). Esse resultado sugere que o desempenho cinético da amostra modificada é menor que da amostra in natura, embora a capacidade de adsorção seja praticamente a mesma. Esse comportamento sugere que a 
amostra modificada não se encontra em condições experimentais que lhe são favoráveis. A partir de um estudo mais detalhado envolvendo o efeito da temperatura bem como o efeito da dosagem do adsorvente deverá sugerir condições operacionais mais adequadas para o emprego do material com maior desempenho.

Tabela 3: Parâmetros da cinética de pseudo-segunda ordem (PSO): constante cinética $\left(\mathrm{k}_{2}\right)$, coeficientes de determinação $\left(\mathbf{R}^{2}\right)$ e capacidade de adsorção $\left(\mathbf{Q}_{\mathrm{e}}\right)$. Concentração do tiofeno: $5 \mathrm{mg} \cdot \mathrm{L}^{-1}$.Massa do adsorvente de $200 \mathrm{mg}$.

\begin{tabular}{|c|c|c|c|c|}
\hline Fibra & $\left.\frac{k_{2}}{(\text { g.mg }}{ }^{-1} \cdot \mathrm{min}^{-1}\right)$ & $\mathbf{R}^{2}$ & $\begin{array}{l}Q_{\mathrm{e}}(\text { cal }) \\
\left(\mathrm{mg}^{-\mathrm{g}^{-1}}\right)\end{array}$ & $\begin{array}{l}Q_{\mathrm{e}}(\exp ) \\
\left(\mathrm{mg}^{-1}\right)\end{array}$ \\
\hline In natura & 0,0178 & 0,9847 & 34,23 & 35,63 \\
\hline Modificada & 0,0228 & 0,9887 & 33,87 & 35,77 \\
\hline
\end{tabular}

A

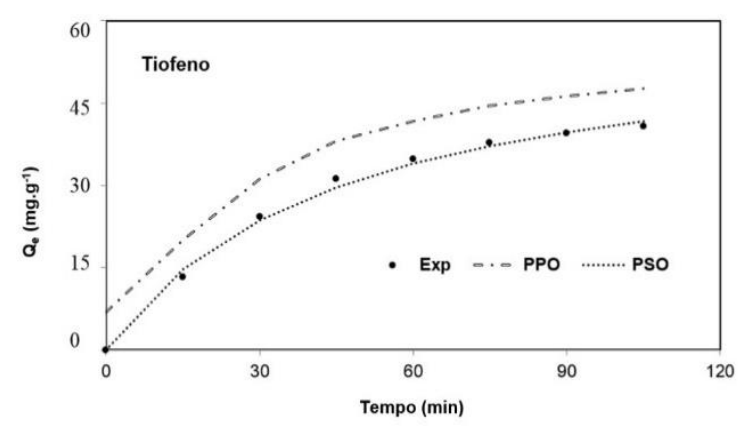

B

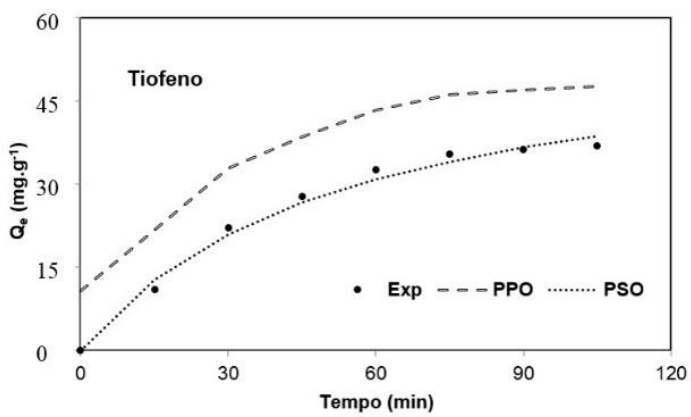

Figura 6: Cinética de adsorção do tiofeno pela fibra in natura, curva A, e pela fibra modificada, curva B. Massa do adsorvente de $200 \mathrm{mg}$; volume de solução $50 \mathrm{~mL}$ e concentração de 5,0 mg. $\mathrm{L}^{-1}$.

A figura 07 mostra as isotermas de adsorção experimental do tiofeno frente à fibra in natura (A) e modificada (B) bem como sua correlação com os diferentes modelos de isotermas estudados. Os parâmetros calculados de todos os modelos são apresentados nas tabelas 04 e 05 , conforme seja o adsorvente a fibra in natura e modificada, respectivamente.

Tabela 4: Parâmetros das isotermas de adsorção do tiofeno pela fibra in natura.

\begin{tabular}{|c|c|c|c|c|c|c|}
\hline Modelo & \multicolumn{2}{|c|}{$\mathbf{Q}_{\text {máx }}$} & \multicolumn{2}{|c|}{$\mathbf{K}_{\mathbf{L}}$} & $\mathbf{R}^{2}$ & SSE \\
\hline Langmuir & \multicolumn{2}{|c|}{37,493} & \multicolumn{2}{|c|}{0,694} & 0,975 & 2,504 \\
\hline \multirow[t]{2}{*}{ Freudlich } & \multicolumn{2}{|c|}{43,081} & \multicolumn{2}{|c|}{0,612} & 0,957 & 4,632 \\
\hline & \multicolumn{2}{|c|}{$\mathbf{Q}_{\text {máx }}$} & $\mathbf{a}$ & b & $\mathbf{R}^{2}$ & SSE \\
\hline \multirow[t]{2}{*}{$\begin{array}{l}\text { Redlich- } \\
\text { Peterson }\end{array}$} & \multicolumn{2}{|c|}{35,127} & 1,679 & 0,231 & 0,979 & 1,510 \\
\hline & $\mathbf{Q}_{\text {máx }}$ & $\mathbf{K}_{\mathbf{T}}$ & & & $\mathbf{R}^{2}$ & SSE \\
\hline Temkin & 43,825 & 3,570 & & & 0,985 & 1,771 \\
\hline
\end{tabular}

Tabela 5: Parâmetros das isotermas de adsorção do tiofeno pela fibra modificada.

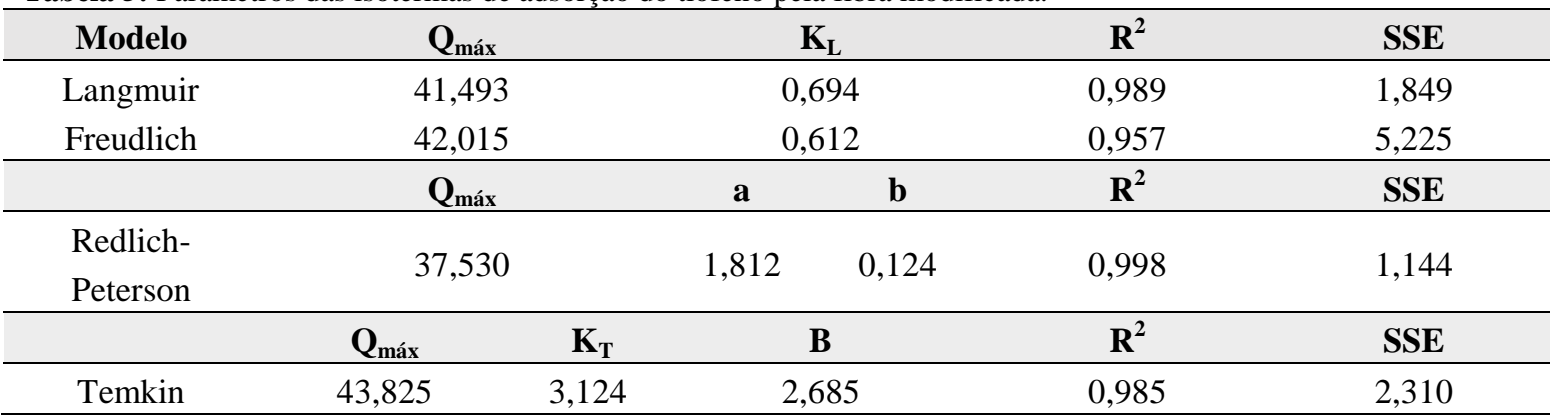



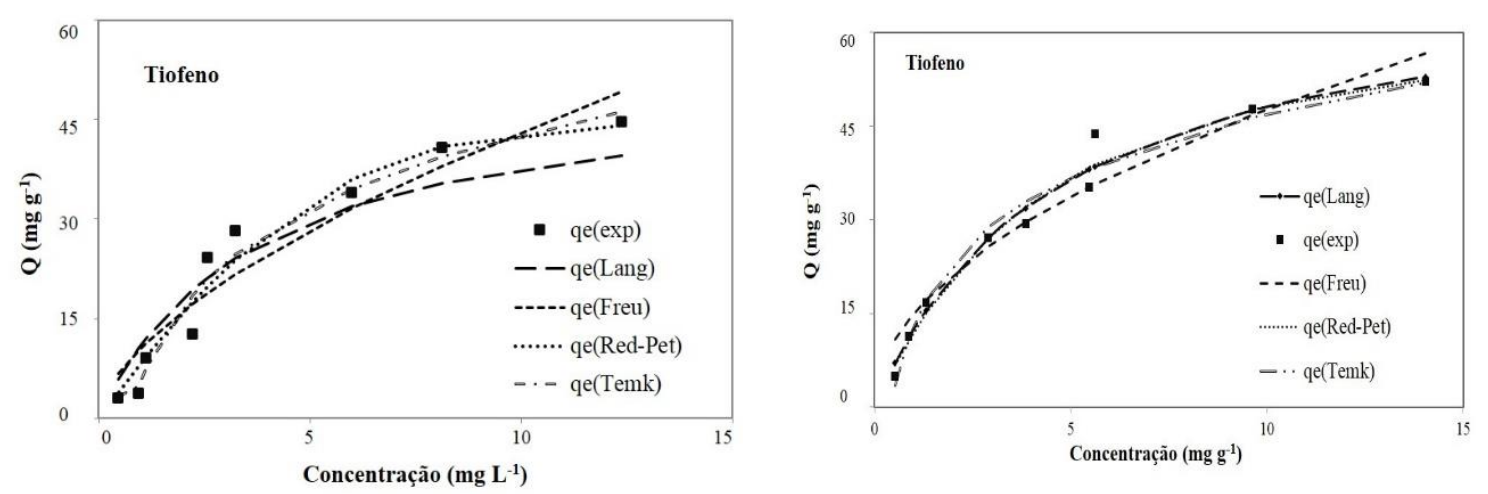

Figura 7: Isotermas de adsorção do tiofeno pela fibra in natura, curva A, fibra modificada, curva B. Massa do adsorvente de $200 \mathrm{mg}$; volume de solução $50 \mathrm{~mL}$ e concentração de 5,0 mg. $\mathrm{L}^{-1}$.

\section{DISCUSSÃO}

Como pode ser observado na figura 03 (TG e DTG), os materiais apresentam tendências em diminuir a massa sistematicamente com a temperatura. No entanto, a fibra in natura revela-se mais instável de modo que aos $180{ }^{\circ} \mathrm{C}$ ocorre uma redução de $60 \%$ da sua massa, parte da qual, conforme, podemos comparar com a fibra modificada, devido à materiais orgânicos extraíveis por solução ácida diluída. Pela DTG determina-se que essa queima ocorre entre as temperaturas de $50{ }^{\circ} \mathrm{C}$ e $100{ }^{\circ} \mathrm{C}$, com pico endotérmico máximo em aproximadamente $60{ }^{\circ} \mathrm{C}$.

De forma comum as duas amostras da fibra de coco, as perdas de massas ocorrem em quatro faixas de temperatura: $20-60{ }^{\circ} \mathrm{C}, 110-140{ }^{\circ} \mathrm{C}, 150-200{ }^{\circ} \mathrm{C}$ e $210-250{ }^{\circ} \mathrm{C}$; as mudanças observadas nessas regiões podem ser atribuídas aos eventos da termodessorção de água e gases, degradação de resquícios de matéria orgânica agregada, degradação da estrutura da fibra e desidroxilações dos grupamentos hidroxílicos, respectivamente [22].

$\mathrm{O}$ resultado gráfico apresentado nas figuras 04 e 05 , perfil $\mathrm{A}$, mostram que a variação no $\mathrm{pH}(3,0$ a $10,0)$ relativo às sucessivas adições de $\mathrm{NaOH} 0,1$ mol. $\mathrm{L}^{-1}$, são tipicamente de um sistema tamponante, ou seja, controlador da variação de acidez/basicidade da solução [23]. Isso está relacionado ao fato da fibra de coco in natura e modificada possuírem grupos químicos funcionais capazes de neutralizar as hidroxilas adicionadas pela base, grupos ácidos de Brönsted e/ou de Lewis. Aplicando a equação 1, que quantifica o total de cargas protônicas na superfície dos materiais em função do $\mathrm{pH}$ do sobrenadante, obtém-se o gráfico apresentado nas figuras 04 e 05 , perfil B.

Percebe-se que ao aumentar o pH, a concentração das cargas protônicas na fibra do coco in natura diminui, isto ocorre porque os íons hidrogênios estão sendo utilizados para controlar as hidroxilas da base adicionada. Até aproximadamente pH igual a 5,0 (na fibra in natura) e 4,3 (na fibra modificada) a quantidade de carga de prótons na superfície apresenta valores positivos devido à existência de grupos doadores de prótons, os sítios ácidos de Brönsted. Por outro lado, acima desses valores de $\mathrm{pH}$, o controle da basicidade é realizado por grupos ácido de Lewis da fibra, pois embora apresente carga protônicas superficiais negativas, ainda exerce efeito tamponante, conforme discussão anterior.

A partir dos resultados apresentados na tabela 01, o teste padrão mostrou que a fibra de coco foi capaz de remover em mais de $71 \%$ o conteúdo de tiofeno. Aplicando a equação 02, determinou-se que a capacidade de adsorção (Qe) está acima de 35,0 mg. ${ }^{-1}$. Em outros estudos utilizando matrizes adsorventes derivados da fibra de coco, os resultados para a capacidade de adsorção variam com a natureza do analito e as condições dos experimentos, conforme dados apresentados na tabela 06. Quando se estuda o carbono ativado obtido da fibra do coco por tratamento térmico, a capacidade de adsorção supera os $100 \mathrm{mg} \cdot \mathrm{g}^{-1}$ na remoção dos compostos carbofurano e fenol dissolvidos em solução aquosa. Contudo, nas pesquisas com fibra in natura para tratamento de efluentes contendo corantes, naftaleno e as espécies químicas inorgânicas como cromo (IV) e mercúrio elementar, os resultados com tiofeno obtidos nesse trabalho mostram valores maiores de capacidade de adsorção. 
Tabela 6: Valores comparativos de capacidade de adsorção, Qe, de adsorventes derivados da fibra do coco, em diversas condições e de diferentes analitos.

\begin{tabular}{|c|c|c|c|c|}
\hline Adsorvente & Analito & $\mathbf{Q e}_{\text {máx }}\left(\mathrm{mg.g}^{-1}\right)$ & Condições** & Referência \\
\hline Fibra do coco & Tiofeno & 35,60 & 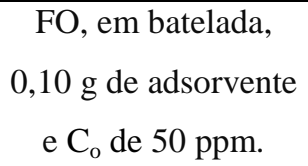 & Nesse estudo \\
\hline $\begin{array}{c}\text { *Carbono ativa- } \\
\text { do }\end{array}$ & Carbofurano & 150,00 & $\begin{array}{c}\text { FA, em batelada, } \\
0,20 \text { g adsorvente e } \\
\text { C }_{\mathrm{o}} \text { varáveis }\end{array}$ & {$[24]$} \\
\hline
\end{tabular}

\begin{tabular}{|c|c|c|c|c|}
\hline \multirow{2}{*}{$\begin{array}{c}\text { *Carbono ativa- } \\
\text { do }\end{array}$} & \multirow[b]{2}{*}{ Fenol } & \multicolumn{3}{|c|}{ FA, em batelada, 100} \\
\hline & & 120,00 & $\begin{array}{c}\mathrm{g} \text { adsorvente e } \mathrm{C}_{\mathrm{o}} \\
\text { varáveis. }\end{array}$ & [13] \\
\hline & Azul de metileno & 25,00 & FA, em batelada, & \\
\hline Fibra do coco & Amarelo direto & 6,00 & $\begin{array}{c}0,05 \mathrm{mg} \text { de adsorven- } \\
\text { te e } \mathrm{C}_{\mathrm{o}} \text { variáveis }\end{array}$ & [15] \\
\hline Fibra do coco & Naftaleno & 5,85 & $\begin{array}{c}\text { FA, em batelada, } \\
0,015 \mathrm{~g} \text { de adsorven- } \\
\text { te e } \mathrm{C}_{\mathrm{o}} \text { de } 100 \mathrm{ppm} \text {. }\end{array}$ & [25] \\
\hline Fibra do coco & Cromo (VI) & 4,50 & $\begin{array}{l}\text { FA, em batelada, } 10 \\
\text { g de adsorvente e } C_{o} \\
\text { de } 100 \text { ppm. }\end{array}$ & [14] \\
\hline Fibra do coco & Mercúrio $\left(\mathrm{Hg}^{0}\right)$ & 0,96 & $\begin{array}{c}\text { FA, em batelada, } \\
0,50 \mathrm{~g} \text { de adsorvente } \\
\text { e } \mathrm{C}_{\mathrm{o}} \text { variáveis }\end{array}$ & [26] \\
\hline
\end{tabular}

*Obtido da casca do coco

** FO = Fase Orgânica; FA = Fase Aquosa; fl = fluxo; $\mathrm{C}_{\mathrm{o}}=$ Concentração inicial;

Pelo estudo de cinética, resultados apresentados nas tabelas 02 e 03, e figura 06, percebe-se que a diferença entre os valores de capacidade de adsorção calculados, $Q_{e}(\mathrm{cal})$, e experimentais $\left(\mathrm{Q}_{\mathrm{e}}, \mathrm{exp}\right.$,) é menor quando se usa o modelo de pseudo-segunda ordem para representar a cinética de adsorção do tiofeno na fibra de coco, seja pelo sistema contendo a o adsorvente in natura ou modificado, tabela 03.

Comparando-se o valor de $\mathrm{k}_{2}$ da tabela 03, observa-se que $\mathrm{k}_{2}$ (amostra in natura) $<\mathrm{k}_{2}$ (amostra modificada). Esse resultado sugere que o desempenho cinético da amostra modificada é menor que da amostra in natura, embora a capacidade de adsorção seja praticamente a mesma. Esse comportamento sugere que a amostra modificada não se encontra em condições experimentais que lhe são favoráveis.

O modelo cinético de pseudo-segunda ordem descreve que a interação do analito-adsorvente seja equiparada a uma quimissorção [27]. Sendo a estrutura atômica da fibra de coco, material celulósico, constituído de vários grupos hidroxílicos, esses devem ser os principais sítios de ligação com o analito, o tiofeno. Mesmo com a modificação química realizada, essencialmente, tratamento com ácido clorídrico e incorporação de níquel, espera-se que a ligação adsorvente-adsorbato se intensifica em função da capacidade de formar complexo de coordenação entre o níquel e o enxofre do tiofeno [28]. Em qualquer das matrizes adsorventes, in natura e modificada, o modelo de pseudo-segunda ordem é apropriado para descrever essa cinética de adsorção. As curvas mostradas na figura 06, relacionando a quantidade adsorvida no equilíbrio $\left(\mathrm{Q}_{\mathrm{e}}\right)$ versus tempo (t) de adsorção, reforçam ainda mais a afirmação de que o modelo de pseudo-segunda ordem consegue descrever satisfatoriamente a cinética dessa adsorção. 
O estudo de isoterma de equilíbrio de adsorção é uma importante ferramenta na investigação do processo de adsorção, pois possibilita relacionar a quantidade de analito adsorvido em solução na superfície do adsorvente depois que o equilíbrio é alcançado [29]. Empregou-se o método de análise de regressão nãolinear para estimar os parâmetros dos modelos matemáticos das isotermas de Langmuir, Freundlich, Temkin e Redlich-Peterson. A função soma dos quadrados dos erros (SSE) foi examinada para todos os dados experimentais e os parâmetros dos modelos de isotermas foram determinados considerando o menor valor do erro (SSE). Em cada caso, o ajuste e otimização das funções matemáticas foram realizados com o aplicativo "solver" do software Microsoft Excel [4, 30]. A função erro ESS é representada pela equação 10.

$$
\mathrm{ESS}=\sum_{\mathrm{i}=1}^{\mathrm{p}}\left(\mathrm{Q}_{\mathrm{e}}, \mathrm{cal}-\mathrm{Q}_{\mathrm{e}}, \exp \right)^{2}
$$

De um modo geral, os dados experimentais da adsorção com a fibra in natura, considerando simultaneamente os menores valores de erro (ESS) e os maiores valores do coeficiente de determinação $\left(\mathrm{R}^{2}\right)$, podem ser melhor correlacionados com os modelos matemáticos que definem as isotermas de Redlich-Peterson e Temkin [30] . Enquanto o modelo Temkin, que considera que o calor de adsorção diminui linearmente com a cobertura da superfície do adsorvente em função das interações indiretas entre as moléculas adsorvidas na superfície do sólido [31], o de Redlich-Peterson preconiza a heterogeneidade na superfície de adsorção, que são compatíveis com a estrutura química do adsorvente, heterogeneidades nos sítios de adsorção com possíveis formação de ligação química.

Uma vez que a constante B, de Temkin, está relacionada ao calor de adsorção (b), conforme a equação 07. Seus valores apresentados nas tabela 04 e 05 mostram que o calor de adsorção do tiofeno com a matriz modificada $(\mathrm{B}=2,685)$ é maior que o da fibra in natura $(\mathrm{B}=1,469)$, possivelmente influenciado pela formação de ligação Níquel - Enxofre [28].

De um modo geral, considerando as bases teóricas dos modelos, detém-se que a adsorção do tiofeno na fibra modificada ocorre de acordo com as condições assumidas por ambos os modelos, ou seja, que adsorção é um fenômeno de monocamada (modelo de Langmuir), mas com sítios de adsorção não idênticos (modelo de Redlich-Peterson) resultando em diferentes afinidades entre os solutos e o adsorvente.

Esses resultados indicam que os sítios de adsorção na fibra de coco, definem que primeira camada de moléculas adsorvidas, são motivados pelas interações de hidrogênio dos grupamentos hidroxílicos da matriz celulósica e o enxofre do tiofeno. Uma outra possível interação, deve ocorrer quando o níquel é incorporado à matriz, gerando ainda mais heterogeneidade superficial de adsorção, justificando a correlação dos dados experimentais com o modelo de Redlich-Peterson.

\section{CONCLUSÕES}

A pesquisa mostra que a fibra de coco, seja na sua forma natural quanto modificada com ácidos e impregnada com sal de níquel, pode ser utilizada na remoção do composto tiofeno, um dos componentes encontrados na gasolina. Sua capacidade de adsorção é superior $35,0 \mathrm{mg} \cdot \mathrm{g}^{-1}$, com cinética de adsorção tipicamente de pseudo-segunda ordem e equilíbrio de adsorção podendo ser representado pelo modelo de Redlich-Peterson, que são característicos para adsorventes que apresentam heterogeneidade superficial em seus sítios de adsorção.

\section{AGRADECIMENTOS}

Os autores agradecem aos colaboradores do Grupo de Analítica e Materiais (GAMa) do IFCE - Campus Quixadá, ao Laboratório de Analítica do IFCE pela realização dos experimentos e análises, ao Laboratório de Química Inorgânica da UFC pelas análises térmicas e ao IFCE pelo apoio estrutural além da CAPES, CNPq e FUNCAP pelo incentivo financeiro.

\section{BIBLIOGRAFIA}

[1] SERRANO, D.P., SANZ, R., PIZZARO, P., et al., "Hierarchical TS-1 zeolite as na eficiente catalyst for oxidative desulphurization of hydrocarbon fractions", Applied Catalysis B: Environmental, v. 146, pp. 35-42, Mar. 2014.

[2] NORMAS BRASIL. http://www.normasbrasil.com.br/norma/?id=261162 Acessado em 02/02/2018.

[3] MELO, P.O.de, Determinação de enxofre em petróleo pesado resíduo de vácuo e coque por ICP OES após combustão iniciada por microondas em sistema fechado, Dissertação de M. Sc., UFSM., Santa Maria, RS, 2012. 
[4] NASCIMENTO, R.F.do, LIMA, A.C.A., VIDAL, C.B., et al., Adsorção: Aspectos teóricos e aplicações ambientais. $1^{\mathrm{a}}$ ed. Fortaleza, Imprensa Universitária. 2014.

[5] KAVITHA, D., NAMASIVAYAM, C., "Experimental and kinetic studies on methylene blue adsorption by coir pith carbon", Bioresource Technology, v. 98, n. 1, pp. 14-21, Jan. 2007.

[6] MALL, I. D., SRISVATAVA, V.C., AGARWAL, N.K., "Adsorptive removal of Auramine-O: Kinetic and equilibrium study”, Journal of Hazardous Materials, v. 143, pp. 386 - 395, May. 2007.

[7] BABICH, I.V. e MOULIJN, J.A., "Science and technology of novel processes for deep desulfurization of oil refinery streams: a review", Fuel, v. 82, n. 6, pp. 607-631, Apr. 2003.

[8] RODRIGUES, A.E., PEREIRA, C., MINCEVA, M., et al., "Principles, Design and Process Applications", In: Elsevier Inc., Simulated Moving Bed Technology, $1^{\text {a }}$ Ed., Chapter 1, Waltham, USA, ButterworthHeinemann, 2015.

[9] ATKINS, P.W., Físico - Química, 5ª Ed., Rio de Janeiro, LTC, Editor. 1997.

[10] LEE, S.-H. e YANG, J.-W., "Removal of Copper in Aqueous Solution by Apple Wastes", Separation Science Technology, v. 32, n. 8, Aug. 1997.

[11] FULAZZAKY, M.A., "Determining the resistance of mass transfer for adsorption of the surfactants onto granular activated carbons from hydrodynamic column", Chemical Engineering Journal, v.166, n.3, pp. 832840, Feb. 2011.

[12] ZHAO, X., ZENG, X., QIN, Y., et al., "An experimental and theoretical study of the adsorption removal of toluene and chlorobenzene on coconut shell derived carbon", Chemosphere, v. 206, n. in progress, pp. 285-292, Sep. 2018.

[13] KARRI, R.R., SAHU, J.N., JAYAKUMAR, N.S., “Optimal isotherm parameters for phenol adsorption from aqueous solutions onto coconut shell based activated carbon: Error analysis of linear and non-linear methods”, Journal of the Taiwan Institute of Chemical Engineers, v. 80, n. 10, pp. 472-487, Nov. 2017.

[14] CHWASTOWSKI, J., STARON, P., KOLOCZEK, H., et al., "Adsorption of hexavalent chromium from aqueous solutions using Canadian peat and coconut fiber”, Journal of Molecular Liquids, v. 248, n. 24, pp. 981-989, Dec. 2017.

[15] ALJEBOREE, A.M., ALSHIRIFI, A.N. e ALKAIM, A.F., "Kinetics and equilibrium study for the adsorption of textile dyes on coconut shell activated carbon", Arabian Journal of Chemistry, v. 10, n. 2, pp. S3381-S3393, May. 2017.

[16] MRAD, I., GHORBEL, A., TICHIT, D., et al., "Optimisation of the preparation of an Al-pillared clay: thermal stability and surface acidity”, Applied Clay Science, v. 12, n. 4, pp. 349-364, Jun. 1997.

[17] FOO, K.Y. e HAMEED, B.H., "Insights into the modeling of adsorption isotherm systems", Chemical Engineering Journal, V. 156, n. 1, pp. 2-10, Jan. 2010.

[18] WIBOWO, N., SETYADHI, L., WIBOWO, D., et al., “Adsorption of benzene and toluene from aqueous solutions onto activated carbon and its acid and heat treated forms: Influence of surface chemistry on adsorption", Journal of Hazardous Materials, v. 146, n. (1-2), pp. 237 - 242, Jul. 2007.

[19] SHENG, G.D., SHAO, D.D., REN, X.M., et al., "Kinetics and thermodynamics of adsorption of ionizable aromatic compounds from aqueous solutions by as-prepared and oxidized multiwalled carbon nanotubes”, Journal of Hazardous Materials, v. 178, n. (1-3), pp. 505-516, Jun. 2010.

[20] MONIER, M., AYAD, D.M., WEI, Y., et al., “Adsorption of $\mathrm{Cu}(\mathrm{II}), \mathrm{Co}(\mathrm{II})$ and Ni(II) ions by modified magnetic chitosan chelating resin", Journal of Hazardous Materials, v. 177, n. (1-3), pp. 962 - 970, May. 2010.

[21] AIVALIOTI, M. VAMVASAKIS, L., GIDARAKOS, E., "BTEX and MTBE adsorption onto raw and thermally modified diatomite", Journal of Hazardous Materials, v. 178, n. (1-3), pp. 136-143, Jun. 2010.

[22] WU, H.-Y., LIAO, C.-H., PAN, Y.-C., et al., "Synthesis and characterization of cubic thiolfunctionalized periodic mesoporous organosilicas as effective mercury ion adsorbents", Microporous and Mesoporous Materials, v. 119, n. (1-3), pp. 109-116, Mar. 2009.

[23] SKOOG, D.A., WEST, D.M., HOLLER, F. J., et al., Fundamentos de Química Analítica. 8a ed., São Paulo, CENGAGE, 2005. 
[24] NJOKU, V.O., SLAM, M.A., ASIF, M., et al., "Preparation of mesoporous activated carbon from coconut frond for the adsorption of carbofuran insecticide", Journal of Analytical and Applied Pyrolysis, v. 110, n. 6, pp. 172-180, Nov. 2014.

[25] LUIS-ZARATE, V.H., RODRIGUEZ-HERNANDEZ, M.C., ALATRISTE-MONDRAGON, F., et al., "Coconut endocarp and mesocarp as both biosorbents of dissolved hydrocarbons in fuel spills and as a power source when exhausted”, Journal of Environmental Management, v. 211, n. 7, p. 103-111, Apr, 2018.

[26] JOHARI, K., SAMAN, N., SONG, S.T., et al., "Adsorption enhancement of elemental mercury by various surface modified coconut husk as eco-friendly low-cost adsorbents", International Biodeterioration \& Biodegradation, v. 109, n.1, pp. 45-52, Apr. 2016.

[27] BELAID, K.D., KACHA, S., KAMECHE, M., et al., "Adsorption kinetics of some textile dyes onto granular activated carbono”, Journal of Environmental Chemical Engineering, v. 1, n.3, pp. 496-503, 2013.

[28] TYAGI, P., CHANDRA, S., SARASWAT, B.S., et al., "Ni(II) and Zn(II) complexes of 2-((thiophen-2ylmethylene)amino)benzamide: Synthesis, spectroscopic characterization, thermal, DFT and anticancer activities", Spectrochimica Acta Part A: Molecular and Biomolecular Spectroscopy, v. 134, pp. 200-209, 2015.

[29] COONEY, D.O., Adsorption design for wasterwater treatment, $1^{\mathrm{a}}$ Ed, London, ENG., Lewis Publishers, 1999.

[30] MOURA, C.P., VIDAL, C.B., BARROS, A.L., et al., “Adsorption of BTX (benzene, toluene, o-xylene, and p-xylene) from aqueous solutions by modified periodic mesoporous organosilica", Journal of Colloid and Interface Science, v. 363, n. 2, pp. 626-634, Nov. 2011.

[31] KUMAR, P.S., RAMALINGAM, S., SENTHAMARAI, C., et al., "Adsorption of dye from aqueous solution by cashew nut shell: Studies on equilibrium isotherm, kinetics and thermodynamics of interactions", Desalination, v. 261, n. (1-2), pp. 52-60, OCT. 2010.

\section{ORCID}

Jaqueline de Lima Nascimento

https://orcid.org/0000-0003-3728-3350

Guilherme Augusto Magalhães Júnior

https://orcid.org/0000-0003-1083-5434

Rafael Ribeiro Portela

https://orcid.org/0000-0003-3359-0089

Vicente de Oliveira Sousa Neto

https://orcid.org/0000-0003-4358-2293

Patrícia Marques Carneiro Buarque

https://orcid.org/0000-0002-0290-0489

Mayara de Sousa Oliveira

https://orcid.org/0000-0002-5830-1979

Cícero Pessoa de Moura

https://orcid.org/0000-0002-3324-7339 